\title{
Discovery of a Disrupting Open Cluster Far into the Milky Way Halo: A Recent Star Formation Event in the Leading Arm of the Magellanic Stream?
}

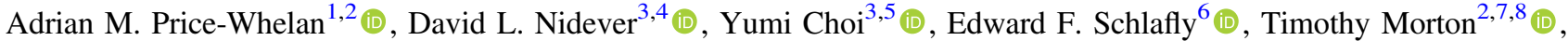 \\ Sergey E. Koposov ${ }^{9,10}$ (10), and Vasily Belokurov ${ }^{8,10}$ (i) \\ ${ }^{1}$ Center for Computational Astrophysics, Flatiron Institute, Simons Foundation, 162 Fifth Avenue, New York, NY 10010, USA; aprice-whelan@ @latironinstitute.org \\ ${ }^{2}$ Department of Astrophysical Sciences, Princeton University, 4 Ivy Lane, Princeton, NJ 08544, USA \\ ${ }^{3}$ Department of Physics, Montana State University, P.O. Box 173840, Bozeman, MT 59717, USA \\ ${ }^{4}$ National Optical Astronomy Observatory, 950 North Cherry Avenue, Tucson, AZ 85719, USA \\ ${ }^{5}$ Steward Observatory, University of Arizona, 933 North Cherry Avenue, Tucson AZ, 85721, USA \\ ${ }^{6}$ Lawrence Berkeley National Laboratory, One Cyclotron Road, Berkeley, CA 94720, USA \\ ${ }^{7}$ Department of Astronomy, University of Florida, 211 Bryant Space Science Center, P.O. Box 112055, Gainesville, FL $32611-2055$ USA \\ ${ }^{8}$ Center for Computational Astrophysics, Flatiron Institute, 162 5th Avenue, New York, NY 10010, USA \\ ${ }^{9}$ McWilliams Center for Cosmology, Carnegie Mellon University, 5000 Forbes Avenue, Pittsburgh, PA 15213, USA \\ ${ }^{10}$ Institute of Astronomy, University of Cambridge, Madingley Road, Cambridge CB3 OHA, UK \\ Received 2018 November 14; revised 2019 September 25; accepted 2019 October 4; published 2019 December 5
}

\begin{abstract}
We report the discovery of a young $(\tau \sim 117 \mathrm{Myr})$, low-mass $\left(M \sim 1200 M_{\odot}\right)$, metal-poor $([\mathrm{Fe} / \mathrm{H}] \sim-1.14)$ stellar association at a heliocentric distance $D \approx 28.7 \mathrm{kpc}$, placing it far into the Milky Way (MW) halo. At its present Galactocentric position $(R, z) \sim(23,15) \mathrm{kpc}$, the association is (on the sky) near the leading arm of the gas stream emanating from the Magellanic Cloud system, but is located $\approx 60^{\circ}$ from the Large Magellanic Cloud center on the other side of the MW disk. If the cluster is colocated with H I gas in the stream, we directly measure the distance to the leading arm of the Magellanic stream. The measured distance is inconsistent with Magellanic stream model predictions that do not account for ram pressure and gas interaction with the MW disk. The estimated age of the cluster is consistent with the time of last passage of the leading arm gas through the Galactic midplane; we therefore speculate that this star formation event was triggered by its last disk midplane passage. Most details of this idea remain a puzzle: the Magellanic stream has low column density, the MW disk at large radii has low gas density, and the relative velocity of the leading arm and MW gas is large. However it formed, the discovery of a young stellar cluster in the MW halo presents an interesting opportunity for study. This cluster was discovered with Gaia astrometry and photometry alone, but follow-up DECam photometry was crucial for measuring its properties.
\end{abstract}

Key words: Galaxy: halo - open clusters and associations: general - stars: formation - surveys

Supporting material: FITS files

\section{Introduction}

The distant stellar halo of the Milky Way (i.e., far from the disk midplane, $|z| \gtrsim 5 \mathrm{kpc}$ ) is typically characterized by its old $(10 \mathrm{Gyr})$, metal-poor $([\mathrm{Fe} / \mathrm{H}] \approx-1.5)$ stellar population. The typical old age of the stellar halo is understood as a signature of the dominant (in stellar mass) progenitor systems that were accreted early on in the formation of the Galaxy (massive dwarf galaxies; Deason et al. 2015; Fiorentino et al. 2015). It is thought that these systems came in with significant gas reservoirs, but were quenched and stripped through collisional processes that heated and dispersed the gas (e.g., Mayer et al. 2006), thus preventing immediate star formation in the deposited gas. The Milky Way, however, continues to accrete satellite galaxies, as is evidenced by the prominent stellar stream from the Sagittarius dwarf galaxy (Ibata et al. 1994; Majewski et al. 2003), the presence of the Large and Small Magellanic Clouds (LMC and SMC), and about 50 dwarf satellites within the Galactic halo. While Sagittarius was likely stripped of its neutral gas long ago (Burton \& Lockman 1999; Tepper-García \& Bland-Hawthorn 2018), the LMC-SMC system is associated with $\approx 8 \times 10^{8} M_{\odot}$ of $\mathrm{H} \mathrm{I}$ gas (Brüns et al. 2005), which extends into leading and trailing gas streams (Mathewson et al. 1974; Putman et al. 1998; Brüns et al. 2005; Nidever et al. 2010). Here, we report the first discovery of a young star cluster in the Milky Way halo that appears to be associated with this LMC-SMC gas stream, which suggests that young stars can form from tidally stripped gas during low-mass mergers and may therefore exist throughout the otherwise aging stellar halo.

The Magellanic stream (MS), including gas in the leading arm (LA), is a large stream of predominantly hydrogen gas emanating from the LMC-SMC system that wraps nearly $\approx 200^{\circ}$ around the sky (Mathewson et al. 1974; Putman et al. 1998; Brüns et al. 2005; Nidever et al. 2010) and contains a significant fraction of the total gas mass associated with the LMC-SMC (Brüns et al. 2005). The trailing MS has been studied in great detail by large-area and high-resolution radio sky surveys: Recent surveys have found small-scale structure and gas fragmentation (e.g., Nidever et al. 2008; For et al. 2014) and a large-scale bifurcation, with kinematically (Nidever et al. 2008) and chemically (Fox et al. 2013) distinct "strands" that lead back to the LMC and the SMC. The LA gas has been found to connect to regions of low-column-density gas $\left(N \sim 10^{18}-10^{19} \mathrm{~cm}^{-2}\right)$ on the other side of the Galactic disk (Putman et al. 1998; Nidever et al. 2008) and has been decomposed into distinct gas features named LA I-IV (Brüns et al. 2005; Nidever et al. 2008; Venzmer et al. 2012).

The origin and formation of these LA features is still uncertain. Initial studies of the LA argued that the features closest to the LMC-SMC can be traced back to the SMC (Putman et al. 1998), but outer features of the LMC appear to 
lead directly into the LA I feature (Nidever et al. 2008). Recent chemical abundance measurements along several sight-lines passing through the LA again support an SMC origin for the gas (Fox et al. 2013, 2018; Richter et al. 2018). Whatever the origin of the LA gas, it is clear that tidal stripping by the Milky Way is required to form the LA (Nidever et al. 2008; Besla et al. 2012). However, the LA features deviate from the predicted orbit of the LMC-SMC, implying that ram pressure or interactions the outer Milky Way disk (from the recent disk plane passage) may have removed orbital energy from the LA gas (e.g., Bekki et al. 2008).

The gas in the MS encodes information about the past and future trajectory of the LMC-SMC, and about interactions between this gas and the Milky Way. Combined with recent proper-motion measurements of the LMC (Kallivayalil et al. 2006, 2013) and improved models for the LMC-SMC that suggest they are on their first passage through the Galaxy (Besla et al. 2007, 2010, 2012), several groups have used the MS to constrain properties of both the interaction history of the LMC-SMC and of the dark matter halo of the Milky Way (see the recent review by D'Onghia \& Fox 2016). In general, these models for the formation of the MS rely on past interactions between the LMC and SMC to preprocess the Magellanic gas distribution before infall and eventual stripping by the tidal field of the Milky Way (Besla et al. 2012; Diaz \& Bekki 2012); More recent hydrodynamical simulations of the Magellanic Clouds (MCs) showed that the repeated encounters between the LMC-SMC strip gas both from the SMC and the LMC, and this tidally stripped gas can then create filamentary structures both in the leading and trailing MS (Pardy et al. 2018). Many observational studies (e.g., Olsen et al. 2011; Noël et al. 2013; Mackey et al. 2016; Carrera et al. 2017; Choi et al. 2018a, 2018b; Zivick et al. 2018; Belokurov \& Erkal 2019) of the LMC-SMC themselves will provide strong constraints the interaction history of the MCs.

One critical difficulty in using the MS to further improve models of the LMC-SMC interaction and infall is the lack of distance information along the MS. No significant over-density of stars has been found that is associated with the trailing MS (Guhathakurta \& Reitzel 1998), thus leaving distance and tangential velocity information unknown. While a small number of $\mathrm{OB}$ stars have been found in the vicinity of the LA gas (Casetti-Dinescu et al. 2014; Zhang et al. 2017), their sparsity and concentration near the Galactic plane make it difficult to unambiguously associate them with the MS and not runaway $\mathrm{OB}$ stars from the Milky Way disk.

In this article, we report the discovery of a young stellar cluster at the far edge of the LA II feature $\left(L_{\mathrm{MS}} \sim 65^{\circ}\right)$ that is located far into the Galactic halo $(D \sim 28.7 \mathrm{kpc})$ and therefore plausibly formed from gas in the leading arm of the MS as it crossed the Galactic disk. This provides the first precise distance measurement to the MS leading arm to be compared with simulations of the LMC-SMC in the Milky Way and provides an opportunity to study recent star formation in a unique environment. The discovery of Price-Whelan 1 will enable new modeling efforts that track the infall of the LMCSMC, the tidal stripping of Magellanic gas, and the interaction of this gas with the Milky Way.

In Section 2, we present the initial discovery with Gaia DR2 and follow-up observations with DECam to obtain deeper photometry of the region around the association. In Section 3, we use the Gaia data to measure the kinematics, and DECam photometry to infer the age, metallicity, and distance to PriceWhelan 1 In Section 4, we interpret the inferred stellar population parameters of Price-Whelan 1 and discuss plausible formation scenarios. We conclude in Section 5.

\section{Data}

\subsection{Cluster Discovery with Gaia}

We use astrometric data from the Gaia mission (Gaia Collaboration et al. 2016), data release 2 (DR2; Gaia Collaboration et al. 2018b; Lindegren et al. 2018) to search for distant, comoving multiplets of blue stars. Our original intent was to search for small, distant, comoving groups of blue horizontal branch stars to identify new candidate satellites of the Milky Way. We therefore initially select all stars from Gaia with parallax $\varpi<1$, color $-0.5<(\mathrm{BP}-\mathrm{RP})<0$, G-band magnitude $G<20$, and Galactic latitude $|b|>20^{\circ}$ (see Appendix for the database query). We further exclude stars within a $15^{\circ}$ radius from the $\mathrm{LMC}$, and a $8^{\circ}$ radius from the SMC $-27,895$ stars remain after these cuts. We then crossmatch this catalog to itself with both sky positions and proper motions: we search for pairs of stars that have separations $s<0.5$ and proper-motion differences $|\Delta \mu|<0.5$ mas $\mathrm{yr}^{-1}$. We then combine mutually connected comoving pairs into small groups of stars that are colocated on the sky and comoving in proper motions, and remove groups that have $<4$ members. We cross-match the mean sky positions of the groups to locations of Local Group galaxies (McConnachie 2012) and Milky Way globular clusters (2010 edition; Harris 1996) and filter out all groups that lie within 1 degree of these known objects. After these filters, one group of comoving stars remains at $($ R.A., decl. $) \sim(179,-29)^{\circ}$.

We then query all objects from the Gaia DR2 catalog within a rectangle centered on the nominal position of this group, with a width of $5^{\circ}$ and a height of $5^{\circ}$ in the equatorial (ICRS) coordinate system (see Appendix for the database query). Figure 1 shows the Gaia data for this region: the left panel shows the Gaia color-magnitude diagram, extinction corrected following the procedure used in Danielski et al. (2018) and the coefficients from Gaia Collaboration et al. (2018a), with the blue over-density highlighted by the polygon (blue) and underplotted with a $100 \mathrm{Myr},[\mathrm{Fe} / \mathrm{H}]=-1.1 \mathrm{MIST}$ isochrone (red line; Paxton et al. 2011, 2013, 2015; Choi et al. 2016; Dotter 2016). Surprisingly, this group appears to be a young, distant main sequence, rather than an old population of horizontal branch stars. The middle and right panels of Figure 1 show sky positions and proper motions of all stars in this sky region (gray background density), and only stars in the color-magnitude diagram (CMD) selection polygon (black markers).

The Gaia data reveal the presence of a young, distant, spatially clustered, and comoving stellar over-density-named Price-Whelan 1-but the Gaia photometry is too shallow to resolve anything but the brightest main-sequence stars. The spatial morphology of the cluster is large on the sky, and interestingly substructured with at least two subcomponents (labeled $\mathrm{a}$ and $\mathrm{b}$ in Figure 1) that are indistinguishable in terms of their proper motions and uncertainties. With the Gaia data alone, the distance, age, and metallicity of the cluster cannot be determined, as these quantities are degenerate where the main sequence is nearly vertical. In the next section, we describe deeper DECam imaging obtained over a portion of the cluster. 
Discovery of Price-Whelan 1 with Gaia
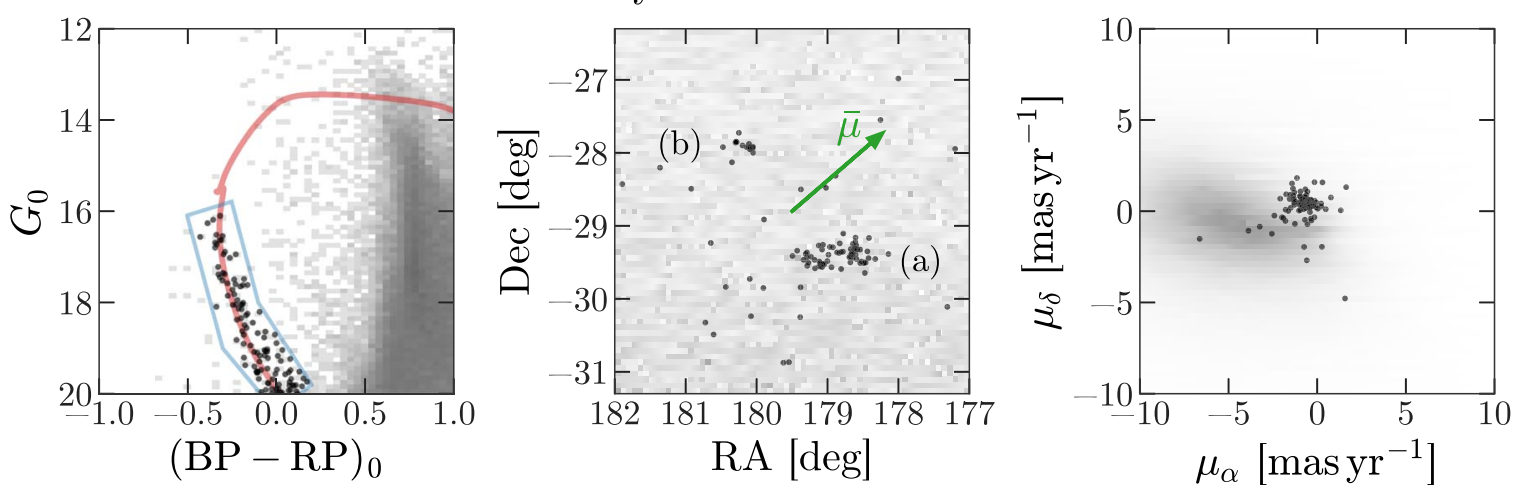

Figure 1. Color-magnitude diagram (CMD), sky positions, and proper motions from Gaia DR2 for the region around $(\alpha, \delta) \sim(179,-29)^{\circ}$. Left panel: Gaia CMD for all sources with $G<20$ and sky position within 3 degrees of $(\alpha, \delta)=(179.5,-28.8)^{\circ}$, extinction corrected following Danielski et al. (2018). The red line shows a $100 \mathrm{Myr},[\mathrm{Fe} / \mathrm{H}]=-1.1 \mathrm{MIST}$ isochrone shifted to a distance of $30 \mathrm{kpc}$. The shaded pixels (gray 2D histogram) show the density of all sources in the CMD for this sky region, and points (black markers) show only sources in the blue selection polygon shown. Middle panel: sky positions for all sources in the shown sky region (gray 2D histogram). The black markers show only sources in the blue selection polygon shown in the CMD (left panel). The arrow (green) indicates the inferred proper-motion direction (Section 3.1). Subcomponents of the cluster ( $a$ and b) are indicated. Right panel: proper motions for all sources in the shown sky region (gray 2D histogram). The black markers, again, show only sources in the blue CMD selection region (left panel).

Table 1

DECam Photometric Transformation

\begin{tabular}{lccc}
\hline \hline Band & Color & Zero-point Term & Color Term \\
\hline$u_{\text {DECam }}$ & $\ldots$ & $27.178 \pm 0.016$ & $\ldots$ \\
$g_{\text {PS1 }}$ & $g_{\text {inst. }}-i_{\text {inst. }}$ & $24.9471 \pm 0.0068$ & $-0.03494 \pm 0.0051$ \\
$i_{\text {PS1 }}$ & $g_{\text {inst. }}-i_{\text {inst. }}$ & $24.7534 \pm 0.0011$ & $-0.07379 \pm 0.0007$ \\
\hline
\end{tabular}

\subsection{Follow Up with DECam}

We obtained DECam $u$-, $g$-, and $i$-band imaging of a single field centered on the "a" spatial component of the cluster (see Figure 1) discovered using the Gaia data (see previous section). Observations were obtained with the Dark Energy Camera (DECam) on the CTIO $4 \mathrm{~m}$ Blanco telescope on UT 2018 May 20 with $3 \times 300 \mathrm{~s}$ exposures in $u$-, $g_{-}$, and $i$-band. The NOAO Community Pipeline (CP; Valdes et al. 2014; F. Valdes et al. 2019, in preparation) InstCal images, which have the instrumental signature removed, were downloaded from the NOAO Archive $^{11}$ for further processing. Forced PSF photometry was performed with the PHOTRED pipeline (Nidever et al. 2017) which uses the DAOPHOT ALLSTAR (Stetson 1987) and ALLFRAME (Stetson 1994) suite of programs.

The instrumental photometry (i.e., $-2.5 \log \left(\mathrm{ADU} \mathrm{s}^{-1}\right)$ was calibrated using Pan-STARRS1 (PS1; Chambers et al. 2016) photometry for $g$ - and $i$-band and with SkyMapper (Wolf et al. 2018) for the $u$-band observations. We use a robust linear fit to cross-matched sources (that appear in both PS1/SkyMapper and our observations) to obtain a zero-point and (for $g$ - and $i$-band) color-term to derive color transformations relating our instrumental DECam photometry to calibrated PS1 grizy and SkyMapper $u$-band photometry (see Table 1 for the derived transformation coefficients). We apply this color transformation to the instrumental photometry to put our photometry on the PS1/DECam system. This calibration enables us to treat the photometry as if it were PS1 ( $g$ - and $i$-band) and DECam ( $u$-band) photometry in the isochrone modeling described below. The robust rms around the linear fit is $0.028 \mathrm{mag}$ for $g$ - and $i$-band and 0.164 mag for the $u$-band; We adopt these rms values as systematic errors that are added in quadrature to the

\footnotetext{
${ }^{11}$ http://archive.noao.edu
}

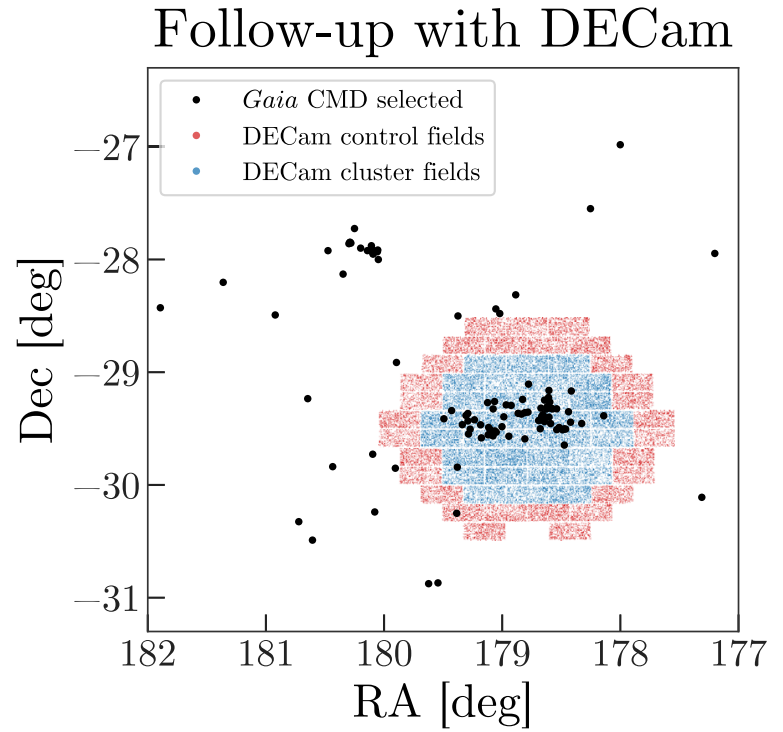

Figure 2. The sky region around Price-Whelan 1, showing the same Gaia CMD-selected sources as in Figure 1 (black markers), and point sources identified from DECam $g$-band follow up (blue and red markers). Red markers show sources in the DECam field used as control sources, and blue markers show sources used as cluster member candidates.

photometric uncertainties for individual sources in the modeling described below. The larger scatter in $u$-band-larger than the uncertainty in the individual photometric measurements-is due to the significant differences between the DECam and SkyMapper $u$-band passbands as well as the $u$-band's sensitivity to both temperature and metallicity. Table 2 contains the photometric measurements for all point sources in the DECam field.

Figure 2 shows the sky positions of the Gaia CMD-selected sources (black markers; see same in middle panel, Figure 1), along with point sources identified in the $g$-band data obtained with DECam: sources in control fields are shown as red markers, and sources in cluster fields are shown as blue markers. We note that the smaller over-density located northeast of the DECam field, component $\mathrm{b}$ (see Figure 1), was not followed up in this work.

Figure 3 shows DECam color-magnitude diagrams for the control and cluster subfields (the magnitudes here are not 
Table 2

DECam Photometry and Sky Positions for Point Sources in the Observed DECam Field

\begin{tabular}{|c|c|c|c|c|c|c|c|c|c|}
\hline \multicolumn{10}{|c|}{ Catalog of DECam Photometry for Point Sources } \\
\hline name & ra & dec & 9 & g_err & $i$ & i_err & control_mask & cluster_mask & member_mask \\
\hline J115311.06-283133.4 & 178.296 & -28.525 & 24.09 & 0.46 & 23.99 & 0.33 & True & False & False \\
\hline J115424.21-283749.6 & 178.600 & -28.630 & 24.61 & 0.25 & 23.83 & 0.28 & True & False & False \\
\hline J115413.93-283343.6 & 178.558 & -28.562 & 25.37 & 0.41 & 22.76 & 0.05 & True & False & False \\
\hline J115419.62-283635.8 & 178.581 & -28.609 & 24.20 & 0.13 & 21.89 & 0.02 & True & False & False \\
\hline & & & & & $\ldots$ & & & & \\
\hline
\end{tabular}

Note. The Boolean columns control_mask and cluster_mask are "True" when a given source is in the control or cluster CCDs, respectively (see Figure 2). The Boolean column member_mask is "True" when the source has a photometric membership probability $>0.5$ of belonging to the cluster stellar population (see Section 3.2).

(This table is available in its entirety in FITS format.)

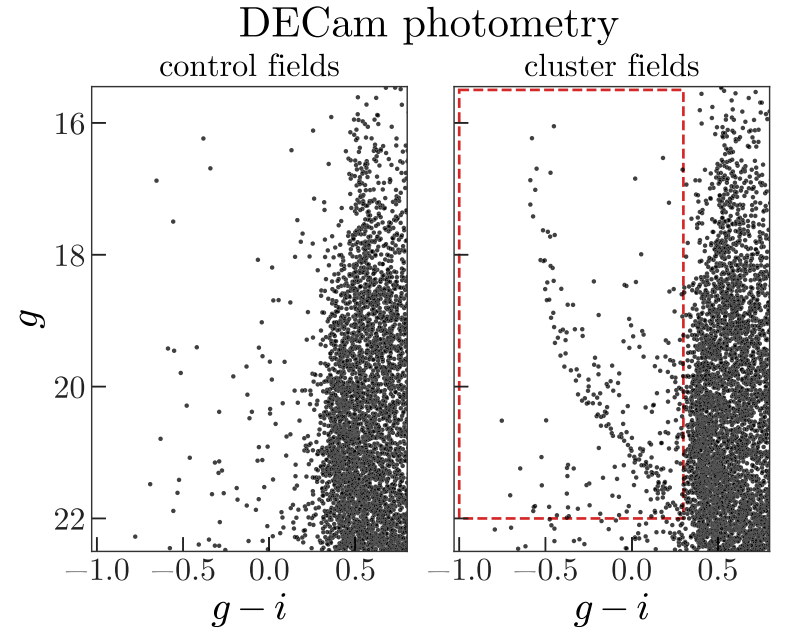

Figure 3. DECam $g-i$ vs. $g$ CMDs for the DECam control fields (left panel) and DECam cluster fields (right panel). Note the prominent young, distant main sequence in the cluster fields: this is the main sequence of Price-Whelan 1. The sources in the dashed (red) rectangle in the right panel are later used to measure the cluster stellar population parameters.

extinction corrected). The blue comoving group, Price-Whelan 1, identified in Section 2.1 using Gaia data alone shows up as a clear young main sequence in the cluster fields (right panel of Figure 3). Later, we use photometry in the subregion of the cluster fields identified by the dashed rectangle to infer the cluster parameters.

\section{Methods}

In the subsections below, we perform two independent analyses of the data available for Price-Whelan 1. First, in Section 3.1, we use astrometric data from Gaia DR2 to determine the mean proper motion of the cluster by modeling the kinematics of the cluster and background sources. Then, in Section 3.2, we use photometric data from DECam to assign membership probabilities to sources and simultaneously measure the cluster stellar population parameters (age, metallicity, distance, etc.). We perform these analyses separately because Gaia DR2 only includes the most massive members of the cluster, but the addition of lower mainsequence members apparent in the DECam photometry provide a much better constraint on the cluster parameters. The
Table 3

Summary of the Measured or Inferred Kinematic and Stellar Population Parameters of the Young Halo Stellar Cluster Price-Whelan 1

\begin{tabular}{lll}
\hline \hline & Inferred properties of Price-Whelan 1 \\
\hline Name & Value & Description \\
$\langle\alpha\rangle$ & 178.8 & R.A. \\
$\langle\delta\rangle$ & $-29{ }^{\circ}$ & Decl. \\
$\langle D\rangle$ & $28.7 \pm 0.4 \mathrm{kpc}$ & Heliocentric distance \\
$\left\langle\mu_{\alpha}\right\rangle$ & $-0.56 \pm 0.04 \mathrm{mas} \mathrm{yr}^{-1}$ & Proper motion in R.A. \\
$\left\langle\mu_{\delta}\right\rangle$ & $0.47 \pm 0.02 \mathrm{mas} \mathrm{yr}^{-1}$ & Proper motion in decl. \\
\hline$\tau$ & $116 \pm 7 \mathrm{Myr}$ & Age \\
$M$ & $1200 M_{\odot}$ & Total stellar mass \\
{$[\mathrm{Fe} / \mathrm{H}]$} & $-1.14 \pm 0.05$ & Metallicity \\
\hline$A_{V}$ & $0.21 \pm 0.02$ & Extinction \\
\hline
\end{tabular}

Note. Formal precisions on all inferred parameters are very small, typically one to a few per cent, but we suspect that systematic errors with the photometry and isochrone models limit the accuracy of these measurements to $\sim 5 \%$.

parameters derived from this analysis are summarized in Table 3.

\subsection{Inferring the Mean Proper Motion with Gaia}

We measure the mean proper motion of Price-Whelan 1 by constructing a probabilistic model of the cluster and background populations using astrometric data from Gaia. We start by selecting all stars with $(\mathrm{BP}-\mathrm{RP})_{0}<0.35$ to remove lowmass and old main-sequence star contamination in the region. Figure 4, left (gray points), shows the sky positions of stars that pass this blue cut in the region around the young cluster. The larger solid-line circle indicates the region we define as the cluster area, and the two smaller dashed-line circles indicate control fields that are combined and used for modeling the background distribution of proper motions. The control fields are designed to, together, have the same total area as the cluster field, and were chosen to have similar latitudes as the cluster field in the MS coordinate system (Nidever et al. 2008). We assume that the background density in the cluster field is equivalent to the average background density of the joint control fields. We do not see any gradients or significant differences in the proper-motion distribution between these two control fields. From visual inspection of the CMDs of the control fields, we do not see any significant clustered 

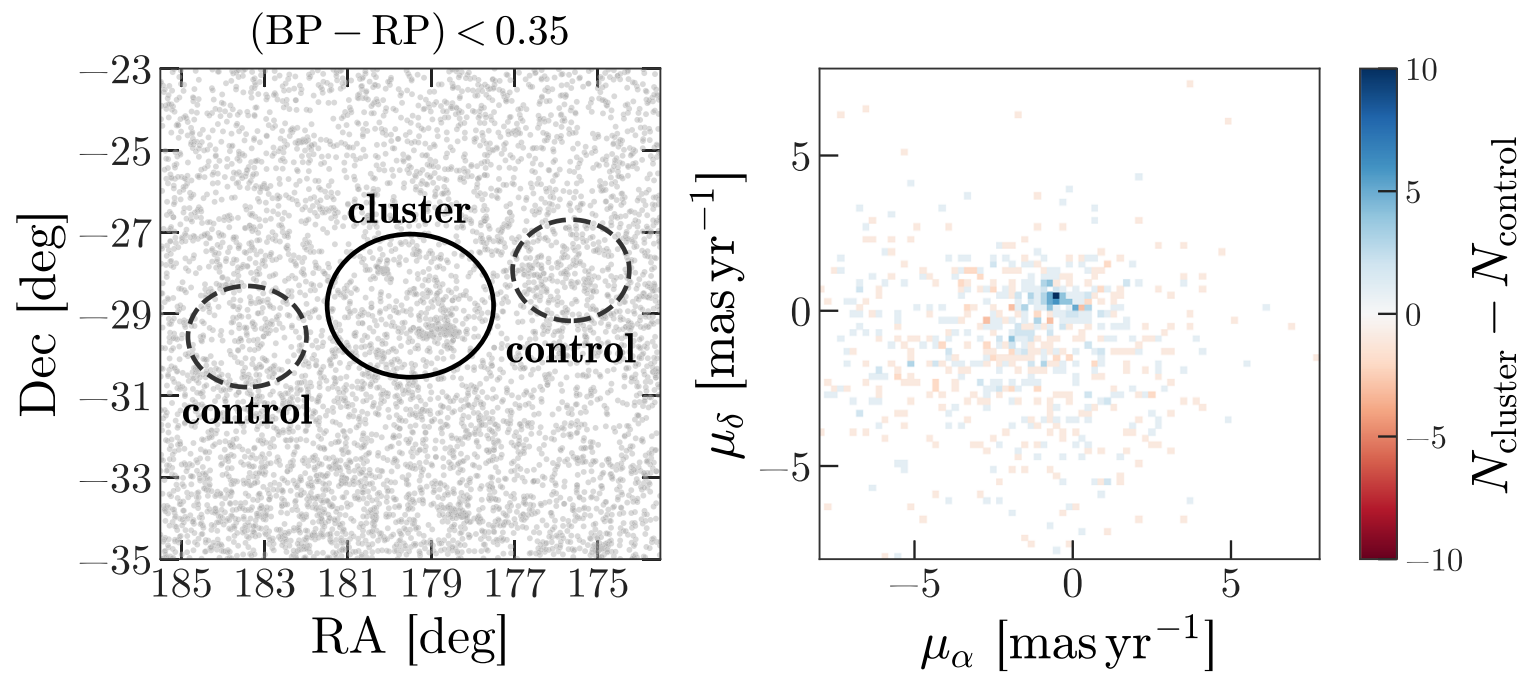

Figure 4. Left panel: sky positions of all Gaia sources in this region with BP $-\mathrm{RP}<0.35$ (gray markers). The circles show the regions used as cluster and control fields in the proper-motion inference (Section 3.1). The control fields, together, have the same area as the cluster field. Right panel: the colored 2D density shows the difference in number of sources per pixel in the cluster field vs. the two control fields (see left panel). The clear over-density in the cluster field (blue) are stars in PriceWhelan 1.

over-density and therefore assume that these fields are dominated by the background stellar density. The right panel of Figure 4 shows the difference of the $2 \mathrm{D}$ proper-motion distributions in the cluster and summed control fields. The distinct over-density of stars near $\left(\mu_{\alpha}, \mu_{\delta}\right) \approx(-0.5,0.5)$ mas $\mathrm{yr}^{-1}$ is the identified comoving association of blue stars. ${ }^{12}$ Note that Figure 4 is only meant as an illustration: The cluster proper motion is determined probabilistically by taking into account the full Gaia covariance matrices for each source, as described below.

To measure the cluster (mean) proper motion, we first construct a model for the error-deconvolved proper-motion distribution in the control fields using "extreme deconvolution" (XD; Bovy et al. 2011) with two Gaussian components. XD takes into account the full error distributions for each propermotion measurement $\boldsymbol{\mu}=\left(\mu_{\alpha}, \mu_{\delta}\right)$, including covariances $\boldsymbol{C}_{\mu}$, provided by Gaia DR2. After running XD on the propermotion distribution of the control fields, we fix the parameters of the density model and use this as the background model for the cluster field. We model the proper-motion distribution in the cluster region using a two-component mixture model with a single, isotropic Gaussian component for the error-deconvolved cluster distribution with mean $\boldsymbol{x}$ and isotropic variance $s^{2}$, and the XD-inferred background model, $p_{\mathrm{XD}}$, for the background component. In detail, taking $f$ to be the fraction of blue stars in this region belonging to the young cluster, and $\tilde{\mu}$ to be the true proper motion for a single star,

$$
\begin{gathered}
p\left(\boldsymbol{\mu} \mid \tilde{\boldsymbol{\mu}}, \boldsymbol{C}_{\mu}\right)=\mathcal{N}\left(\boldsymbol{\mu} \mid \tilde{\boldsymbol{\mu}}, \boldsymbol{C}_{\mu}\right) \\
p(\tilde{\boldsymbol{\mu}} \mid f, \boldsymbol{x}, s)=f p_{\mathrm{cl}}(\tilde{\boldsymbol{\mu}} \mid \boldsymbol{x}, s)+(1-f) p_{\mathrm{XD}}(\tilde{\boldsymbol{\mu}}) \\
p_{\mathrm{cl}}(\tilde{\boldsymbol{\mu}} \mid \boldsymbol{x}, s)=\mathcal{N}\left(\tilde{\boldsymbol{\mu}} \mid \boldsymbol{x}, s^{2} \mathbb{I}\right)
\end{gathered}
$$

where $\mathcal{N}(\cdot \mid \boldsymbol{y}, \boldsymbol{C})$ represents the multidimensional normal distribution with mean $\boldsymbol{y}$ and covariance matrix $\boldsymbol{C}$, and $\mathbb{I}$ is the identity matrix. Because all distributions are Gaussian, the per-star parameters (the true proper motions, $\tilde{\boldsymbol{\mu}}$ ) can be analytically marginalized out so that the per-star likelihood

\footnotetext{
12 Throughout this article, we use $\mu_{\alpha}$ to refer to the proper-motion value provided by Gaia, which includes the $\cos \delta$ term.
}

can be expressed as $p\left(\boldsymbol{\mu} \mid f, \boldsymbol{x}, s, \boldsymbol{C}_{\mu}\right)$. We assume that the measurements for each star, $n$, are independent so that the full likelihood of all $N$ stars given the parameters $(f, \boldsymbol{x}, s)$ is

$$
p\left(\left\{\boldsymbol{\mu}_{n}\right\}_{N} \mid f, \boldsymbol{x}, s,\left\{\boldsymbol{C}_{\mu, n}\right\}_{N}\right)=\prod_{n}^{N} p\left(\boldsymbol{\mu}_{n} \mid f, \boldsymbol{x}, s, \boldsymbol{C}_{\mu, n}\right) .
$$

We use an ensemble Markov Chain Monte Carlo sampler (emcee; Foreman-Mackey et al. 2013b; Goodman \& Weare 2010) to generate posterior samples over the parameters $(f, \boldsymbol{x}, s)$ using the likelihood defined above (Equation (4)), and assuming the following prior probability distributions: uniform over the domain $(-5,5)$ mas $\mathrm{yr}^{-1}$ for each component of $\boldsymbol{x}$, uniform in $f$, and uniform in log-s over the domain $-6<\ln s<4$ (with $s$ in units of mas $\mathrm{yr}^{-1}$ ). We run the sampler with 32 walkers for 256 steps as burn-in, then reset the sampler and run for an additional 512 steps, after which the chains appear converged. We then compute the Gelman-Rubin (Gelman \& Rubin 1992) convergence diagnostic and find that all chains have $R<1.1$. We then downsample the resulting chains by taking every 16 th sample to preserve closerto-independent samples, leaving a total of 1024 samples. We use these samples to estimate the median posterior parameter values and uncertainties. For the young cluster, we find $x=(-0.56$, $0.47) \pm(0.04,0.02)$ mas $\mathrm{yr}^{-1}, \ln s=-3.8 \pm 0.9$, and $f=$ $0.14 \pm 0.02$. The proper-motion dispersion is consistent with zero but is unmeasured: $95 \%$ of the posterior samples have $s<0.09$ mas $\mathrm{yr}^{-1}$, indicating that the observed proper-motion dispersion is consistent with the proper-motion Gaia uncertainties, which have minimum and median values of $\sim 0.09$ mas $\mathrm{yr}^{-1}$ and $\sim 0.4$ mas $\mathrm{yr}^{-1}$, respectively. Table 4 contains a subset of the Gaia DR2 data for stars in the cluster field, with kinematic membership probabilities determined from this modeling procedure.

\subsection{Inferring the Stellar Population Parameters with DECam Photometry}

While the Gaia data provide exquisite astrometric data for the brightest members of Price-Whelan 1, constraints on the stellar population from the Gaia data alone are limited by degeneracies 
Table 4

Select Gaia DR2 Data and Membership Probabilities Computed for All Stars in the Cluster Region (see Figure 4)

\begin{tabular}{|c|c|c|c|c|c|c|c|c|}
\hline \multicolumn{9}{|c|}{ Catalog of Gaia Sources and Kinematic Probability } \\
\hline 3462048793708019200 & 184.799 & -34.971 & 0.09 & -1.61 & 19.79 & 19.915 & 19.61 & 0.00 \\
\hline 3462062670747949312 & 184.426 & -34.816 & -0.56 & -2.77 & 20.33 & 20.363 & 20.06 & 0.00 \\
\hline 3462062709402484224 & 184.407 & -34.809 & -7.50 & -3.16 & 20.67 & 20.437 & 20.09 & 0.01 \\
\hline 3462065904858152832 & 184.705 & -34.783 & 0.91 & -1.95 & 20.64 & 20.625 & 20.37 & 0.12 \\
\hline
\end{tabular}

(This table is available in its entirety in FITS format.)

in the mapping from the color-magnitude diagram to stellar parameters. In addition, uncertainty in the Gaia passbands can cause significant differences in Gaia BP - RP color for very blue sources (Maíz Apellániz \& Weiler 2018). To measure the cluster population parameters, we therefore use the deeper photometry from DECam, which provides a clear view of the main sequence of Price-Whelan 1 down to stellar masses $M \sim 0.9 M_{\odot}$. We note that this analysis and the subsequent discussion only considers subregion (a) of Price-Whelan 1, which dominates the total mass and number of stars in the cluster. Figure 3 shows the DECam $g$ - and $i$-band color-magnitude diagrams for control fields (left) and cluster fields (right) selected from the DECam footprint, once again showing the young main sequence (coherent stellar population in right panel). We use the $g$ - and $i$-band photometry - and $u$-band, though only the brightest $\sim 15$ cluster field members are robustly detected; however for those sources, the $u$-band significantly helps to constrain the extinction for the cluster-for individual stars in a subsection of the cluster field CMD (red dashed outlined region in Figure 3) to infer the stellar population parameters of Price-Whelan 1. To summarize our methodology, we first generate independent posterior samplings over the stellar parameters of each individual source under an interim prior (over age, stellar mass, distance, etc.), then use these individual samplings to construct a Bayesian hierarchical model for a two-component mixture model of the cluster and a background population. This methodology is more robust than conventional isochrone-fitting methods that require by-hand fitting of stellar population parameters and naturally propagates uncertainties in the photometry and cluster membership. If you prefer to skip the details, the results of this modeling are presented in Section 4.1.

In detail, we start by using the isochrones package (Morton 2015) to generate posterior samplings over stellar parameters for each individual source given its photometry and an interim prior. We use the MIST (Paxton et al. 2011, 2013, 2015; Choi et al. 2016; Dotter 2016) isochrone grid, and isochrones automatically performs interpolation between the provided grid of stellar isochrones to predict photometry given a set of stellar parameters. Here, the stellar parameters for each source are its "equal evolutionary point" number EEP (see MIST documentation ${ }^{13}$ ), age $\tau$, metallicity [Fe/H], extinction $A_{V}$, and distance $D$, which can be uniquely mapped to a point in the observed CMD. The likelihood of a given set of these parameters, $\boldsymbol{\theta}=\left(\mathrm{EEP}, \tau,[\mathrm{Fe} / \mathrm{H}], A_{V}, D\right)$, is then computed from the photometry and (assumed Gaussian) photometric uncertainties given the predicted photometry.

\footnotetext{
${ }^{13}$ http://waps.cfa.harvard.edu/MIST/
}

Table 5

Prior Probability Distributions for Each of the Stellar Parameters Defined in Section 3.2, Used for the Independent (Per Star) Posterior Samplings

\begin{tabular}{lcc}
\hline \hline Parameter & Prior & Bounds \\
\hline$\tau$ & uniform & $(10 \mathrm{Myr}, 15 \mathrm{Gyr})$ \\
{$[\mathrm{Fe} / \mathrm{H}]$} & uniform & $(-2,0.5)$ \\
$\mathrm{EEP}$ & (see caption) & $(202,355)$ \\
$A_{V}$ & $\propto A_{V}^{-1}$ & $(0.001,1) \mathrm{mag}$ \\
$D$ & $\propto D^{2}$ & $(1,100) \mathrm{kpc}$ \\
\hline
\end{tabular}

Note. The stellar parameters here are the "equal evolutionary point" number EEP, age $\tau$, metallicity $[\mathrm{Fe} / \mathrm{H}]$, extinction $A_{V}$, and distance $D$. The prior on EEP is computed by calculating the stellar mass, $m$, corresponding to a given point in the isochrone parameter space, $(\mathrm{EEP}, \tau,[\mathrm{Fe} / \mathrm{H}])$, and computing the probability from a Salpeter (Salpeter 1955) mass prior such that $p(\mathrm{EEP})=p(m)\left|\frac{d m}{d \mathrm{EEP}}\right|$, following the prescription used in isochrones (Morton 2015).

To generate posterior samples, we must also specify prior probability distributions over the parameters $\boldsymbol{\theta}$. The priors for each parameter are summarized in Table 5. The bounds on EEP limit the isochrone to evolutionary phases between the zero-age main sequence to the terminal-age main sequence, but this prior is actually computed using the stellar mass computed from the isochrone parameters (see caption of Table 5). The prior and bounds on $A_{V}$ are set to prefer small and reasonable values of extinction for this moderately high Galactic latitude region. The prior on distance assumes a uniform space density of stars.

We find that the photometry for individual sources in the lower main sequence are very poorly constrained in all parameters, and the prior tends to pull the posterior samplings to prefer closer, older stellar parameters. This is a weakness of our methodology for performing the hierarchical inference: Each source is considered independently, even though there is clear structure in the CMD (i.e., Figure 3), and inferring isochronal parameters for individual lower main-sequence stars is a fundamentally degenerate problem. A more correct way to do this would be to infer the stellar parameters of all stars, the cluster hyperparameters, and the background simultaneously. However, for the 417 stars we are using (in the red box in Figure 3), this model would have $\sim 2000$ free parameters if left unmarginalized. We are developing tools to perform star cluster parameter inference in this way (T. Morton et al. 2019, in preparation), but here we adopt a simple hack to allow us to instead perform individual posterior samplings and then combine those samplings into a hierarchical inference. 
Table 6

Prior Probability Distributions for the Hyperparameters $(\boldsymbol{\alpha}, f)$, Used for the Hierarchical Inference of the Cluster Stellar Population Parameters

\begin{tabular}{lcc}
\hline \hline Parameter & Prior & Bounds \\
\hline$\tau^{*}$ & uniform & $(1 \mathrm{Myr}, 1 \mathrm{Gyr})$ \\
{$[\mathrm{Fe} / \mathrm{H}]^{*}$} & uniform & $(-2,0)$ \\
$A_{V}^{*}$ & uniform & $(0,1) \mathrm{mag}$ \\
$D^{*}$ & uniform & $(1,100) \mathrm{kpc}$ \\
$f$ & uniform & $(0,1)$ \\
\hline
\end{tabular}

Note. The cluster parameters here are the cluster age $\tau^{*}$, metallicity $[\mathrm{Fe} / \mathrm{H}]^{*}$, extinction $A_{V}^{*}$, and distance $D^{*}$, and the fraction of stars in the field that belong to the cluster $f$.

The brightest stars in the cluster have very precise DECam photometry and have precise astrometry from Gaia. Given their location in the CMD, these stars must be young. We therefore use the brightest kinematic member of Price-Whelan 1-Gaia source ID 3480046557809199616 - as an "anchor" star: We model every other source in the DECam selection region (i.e., excluding this one) by fitting the photometry of it and the anchor star simultaneously, assuming they have the same age, metallicity, distance, and extinction but different EEP values. Motivated by the possible signature of an (unresolved) binary sequence in the DECam CMD, we add one further piece of complexity to the model by allowing the photometry of each non-anchor source to be fit as an unresolved binary star system. This adds an additional parameter, the unresolved binary mass ratio $q$, to the list of inferred stellar parameters for each source, but, for this work, we ignore the binary companions and implicitly marginalize over $q$ in what follows. The details of this model are handled by the isochrones package.

We generate posterior samplings over the parameters $(\boldsymbol{\theta}, q)$ for each of the 417 sources in the selected region of the DECam CMD using PyMultinest (Feroz \& Hobson 2008; Feroz et al. 2009; Buchner et al. 2014), and store the value of the prior evaluated at the location of each sample. We note that for individual sources, the derived stellar parameters are often very uncertain: For example, the uncertainties on metallicity and distance can be as large as $\sim 0.4$ dex and $\sim 5 \mathrm{kpc}$, respectively. However, we then use these samples and interim prior values to construct our hierarchical model, as described below.

In the hierarchical model, we assume that the stellar parameters of each primary star in the selected region are either drawn from the cluster, or a background (stellar halo) population. For the cluster, we assume that the values are drawn from delta functions in age, metallicity, distance, and extinction, with the centroids of the delta functions $\boldsymbol{\alpha}=\left(\tau^{*},[\mathrm{Fe} / \mathrm{H}]^{*}, D^{*}, A_{V}^{*}\right)$ as hyperparameters of the hierarchical inference- that is, we assume that the cluster is a single stellar population (SSP). We note that we have run the same hierarchical model allowing for Gaussian spreads in age, metallicity, and distance where we simultaneously infer the variances in each cluster parameter, but we have found that the variances are unconstrained, and we therefore instead treat the cluster as an SSP.

For the background model, we assume the same priors as specified in Table 5. The one additional parameter that must be included in this hierarchical model is the mixture weight: The global fraction of sources that are likely cluster members, $f$. To compute the likelihood for the hierarchical model, we use the individual posterior samplings to marginalize over the persource stellar parameters $\boldsymbol{\theta}_{n}$ to compute the marginal likelihood

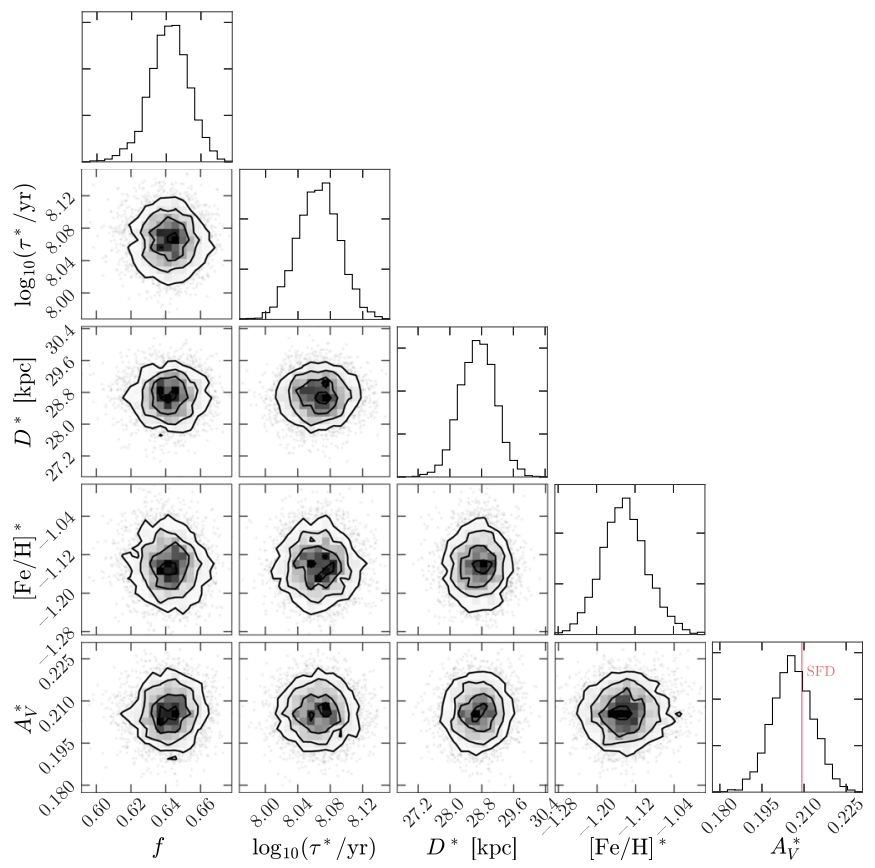

Figure 5. A corner plot showing marginal posterior probability distributions estimated from the the posterior samples generated from the hierarchical inference of the cluster population parameters.

$p\left(\boldsymbol{m}_{n} \mid \boldsymbol{\alpha}, f\right)$, where $\boldsymbol{m}_{n}=(g, i)_{n}$ is the vector of photometric data for source $n$; This likelihood for a single source given a set of hyperparameters $(\boldsymbol{\alpha}, f)$ is then

$$
p\left(\boldsymbol{m}_{n} \mid \boldsymbol{\alpha}, f\right)=\int d \boldsymbol{\theta}_{n} p\left(\boldsymbol{m}_{n} \mid \boldsymbol{\theta}_{n}\right) p\left(\boldsymbol{\theta}_{n} \mid \boldsymbol{\alpha}, f\right) .
$$

We employ the "importance sampling trick" (see, e.g., Appendix of Price-Whelan et al. 2018 or Hogg et al. 2010; Foreman-Mackey et al. 2014 for other examples) to re-write an approximate form for this marginal likelihood as

$$
p\left(\boldsymbol{m}_{n} \mid \boldsymbol{\alpha}, f\right) \approx \frac{\mathcal{Z}_{n}}{K} \sum_{k}^{K} \frac{p\left(\boldsymbol{\theta}_{n k} \mid \boldsymbol{\alpha}, f\right)}{p\left(\boldsymbol{\theta}_{n k} \mid \boldsymbol{\alpha}_{0}, f\right)}
$$

where the index $k$ specifies the index of one of $K$ posterior samples generated from the independent samplings (described above), $\mathcal{Z}_{n}$ is a constant, and the denominator, $p\left(\boldsymbol{\theta}_{n k} \mid \boldsymbol{\alpha}_{0}, f\right)$, are the values of the interim prior used to do the independent samplings. In this work, $N=417$ and we adopt $K=2048$.

With the marginal likelihood (Equation (6)), we then need to specify prior probability distributions for the hyperparameters $(\boldsymbol{\alpha}, f)$, and we can then generate posterior samples for the hyperparameters. We use uniform priors for all of these, as summarized in Table 6. We use emcee (Goodman \& Weare 2010; Foreman-Mackey et al. 2013b) to sample from the posterior probability distribution for the hyperparameters given all of the photometric data,

$$
p\left(\boldsymbol{\alpha}, f \mid\left\{\boldsymbol{m}_{n}\right\}\right) \propto p(\boldsymbol{\alpha}) p(f) \prod_{n}^{N} p\left(\boldsymbol{m}_{n} \mid \boldsymbol{\alpha}, f\right) .
$$

Here we use 64 walkers and run for an initial 128 steps to burn-in the sampler before running for a final 1024 steps. We again compute the Gelman-Rubin (Gelman \& Rubin 1992) convergence diagnostic and find that all chains have $R<1.1$ and are thus likely converged. Figure 5 shows a corner plot with all $1 \mathrm{D}$ and $2 \mathrm{D}$ 
marginal posterior probability distributions estimated from the samples.

\section{Results}

\subsection{Stellar Population and Physical Characteristics}

We use the posterior samples from the hierarchical inference described in Section 3.2 to compute the posterior probabilities that each source in the DECam CMD selection box (red box, Figure 3) is a member of the cluster. Figure 6 shows the DECam photometry for all sources with membership probability $>0.5$ (circle markers). Over-plotted in Figure 6 (blue, solid line) is an MIST isochrone with the median posterior parameters derived from the hierarchical modeling, shifted to the median distance and extincted given the median $A_{V}$ value. Under the assumption that Price-Whelan 1 has a single stellar population, we find that Price-Whelan 1 is indeed young, distant, and metal-poor, with median posterior values and standard deviations of age $\tau=117 \pm 23 \mathrm{Myr}$, distance $D=$ $28.7 \pm 0.5 \mathrm{kpc}$, and metallicity $[\mathrm{Fe} / \mathrm{H}]=-1.14 \pm 0.05$ (see also Table 3 for a summary). We find that the inferred extinction, $A_{V}=0.205 \pm 0.011$, is consistent with the value $A_{V, \mathrm{SFD}} \approx 0.2$ from the (recalibrated) SFD dust map (Schlegel et al. 1998; Schlafly \& Finkbeiner 2011). We note again that, with the DECam photometry, we are limited to studying the subcomponent a of Price-Whelan 1-follow-up imaging of component b (see Figure 1) would enable a similar analysis for the full cluster.

Also plotted in Figure 6 is the equal-mass binary sequence (green, dashed line) computed from the median posterior sample: The abundance of sources between the nominal isochrone and the binary sequence highlights the fact that the cluster may contain a significant number of binary or multiple star systems, but we leave a detailed study of multiplicity to future work. We note that, similar to young open clusters in the Milky Way disk (e.g., Gaia Collaboration et al. 2018a), the bluest stars in the CMD have more apparent scatter than the lower main sequence. This is likely a combination of many things that are not addressed in this work, such as binarity, convective core overshooting (e.g., Yang \& Tian 2017; Johnston et al. 2019), or an intrinsic spread in stellar parameters.

We use the isochrone corresponding to the median posterior sample to estimate the total stellar mass of the cluster. By assuming that the DECam imaging is $100 \%$ complete to stars with $(g-i)<0.3)$ and $g<22$, and by assuming a Kroupa initial mass function (Kroupa 2001), we use the number of observed stars and the isochrone to compute the total mass, $M_{\mathrm{tot}, *} \approx 1200 M_{\odot}$.

The mass and age of Price-Whelan 1 are comparable to Milky Way disk open clusters, but with a much lower metallicity, and a much larger spatial extent. For example, the Pleiades has an age $~ 135$ Myr (Gossage et al. 2018), but a physical size $\sim 5 \mathrm{pc}$. At a distance of $28.7 \mathrm{kpc}$, Price-Whelan 1 spans $\sim 1.5$, corresponding to a physical size $\sim 700-800 \mathrm{pc}$. This is more comparable to (but still larger than) recent star formation sites in the Magellanic bridge (e.g., Mackey et al. 2017), which likely formed as a result of the violent interaction between the MCs. If Price-Whelan 1 formed unbound, but with an initial size comparable to the present size of the Pleiades, this corresponds to an expansion velocity $\sim 6 \mathrm{~km} \mathrm{~s}^{-1}$, which would be detectable with precise radial velocity measurements of stars on either side of Price-Whelan 1.

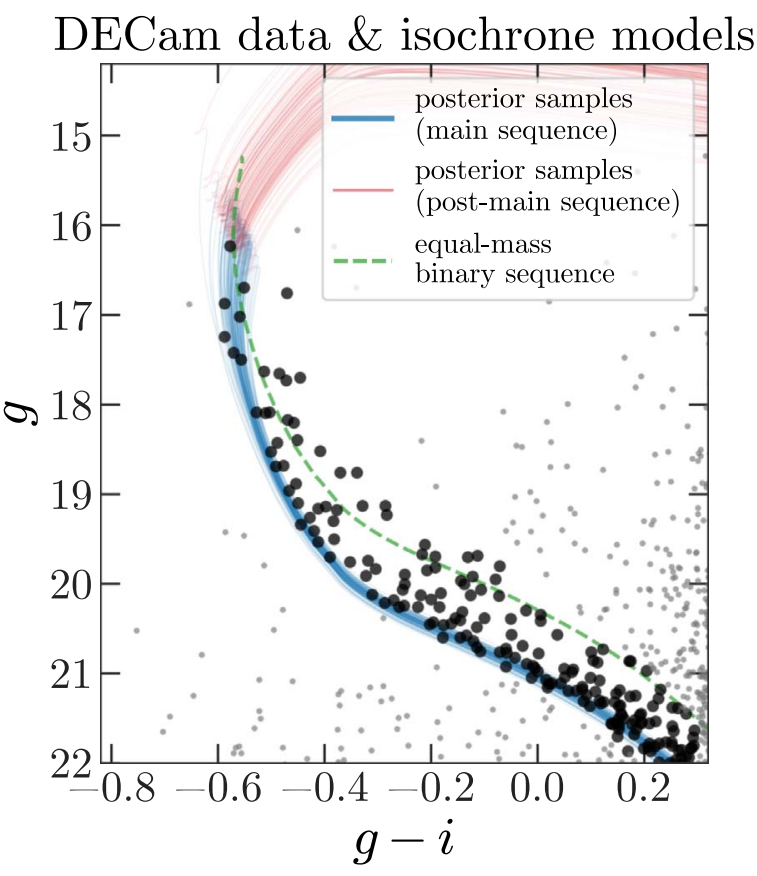

Figure 6. The DECam color-magnitude diagram for sources with probability $>0.5$ of belonging to Price-Whelan 1 (dark, larger markers), and sources with probability $<0.5$ (lighter, smaller markers). The solid line (blue) shows the MIST isochrone for the median posterior sample from the hierarchical inference of the cluster population parameters, shifted to the inferred distance to Price-Whelan 1, and extincted with the inferred extinction. The dashed line (green) shows the same isochrone, shifted $\approx 0.75$ mag brighter, representing the expected location of the equal-mass binary sequence of the cluster.

We caution that the results presented above are conditional on (and likely biased by) the assumption of a single stellar population when modeling the photometry. For example, if the cluster instead has a spread in internal properties (age or metallicity), or significant structure along the line of sight (LOS), the reported mean cluster distance could be biased, and the true mean distance could be up to $\approx 5-6$ kpc closer. Additionally, we do not place any constraints or priors on the binary fraction, and thus the hierarchical model used above may artificially absorb structure along the LOS into a high binary fraction. Future spectroscopic follow up will help to improve interpretation and modeling of the stellar population of Price-Whelan 1.

\subsection{Relation to the Magellanic Stream}

At the sky location and distance of Price-Whelan 1, i.e., well into the Galactic halo, the only plausible gas reservoirs that could have formed a young cluster are the MS, or a previously unknown high velocity cloud (HVC). HVCs are thought to either be accreted and therefore lower metallicity than typical present-day Milky Way gas, or ejected from the Milky Way through a "Galactic fountain"-like process and therefore comparable metallicity to disk gas. Both processes clearly occur: the MS itself is evidence of gas accretion into the Milky Way halo, and the mysterious Smith Cloud (Smith 1963) is a metal-rich $([\mathrm{Fe} / \mathrm{H}] \sim 0.5$ Fox et al. 2016) HVC that plausibly originated from the Galactic disk (e.g., Bregman 1980). Given the low metallicity of the stars in Price-Whelan 1, the gas it formed from was likely extragalactic, as any violent starforming regions in the Milky Way disk that could have driven gas so far out into the halo have, at present-day, significantly higher metallicity than this cluster. 


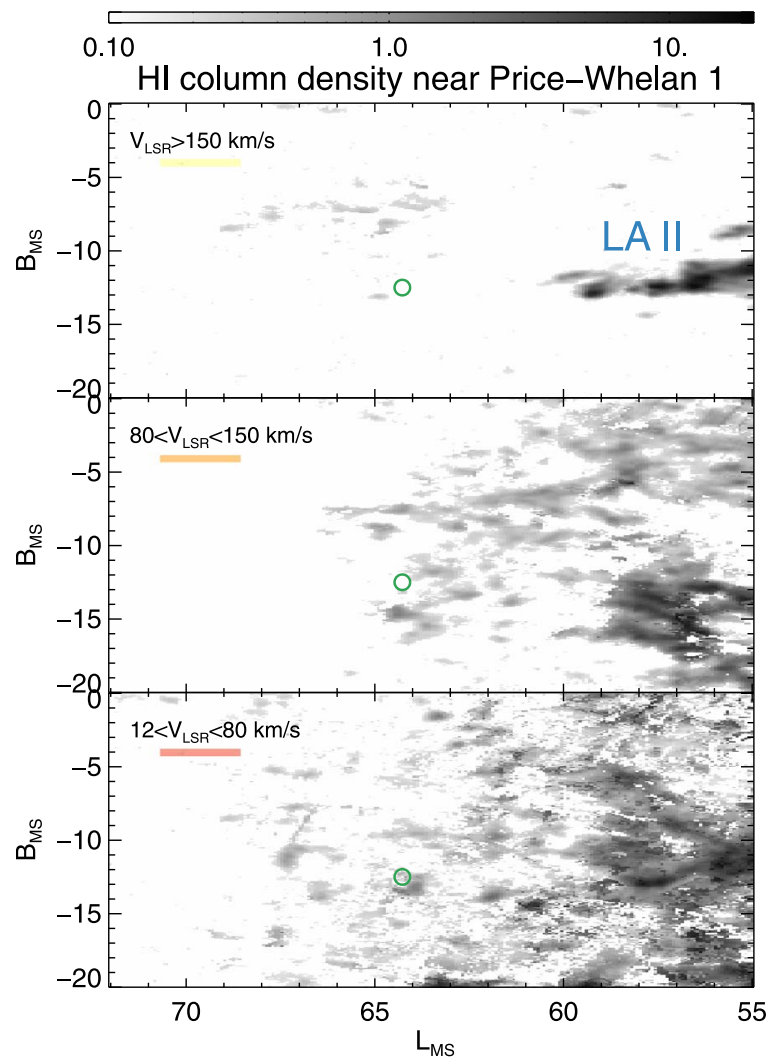

Figure 7. GASS H I column density in the region of LA II, shown in three different velocity slices (corresponding to and indicated in each panel). The colored rectangles under each velocity label correspond to the colored ranges in Figure 8. The coordinate system is the MS spherical coordinate system defined in Nidever et al. (2008), and the units of $L_{\mathrm{MS}}$ and $B_{\mathrm{MS}}$ are degrees. The horizontal colorbar shows the column density of $\mathrm{HI}$ in units of $10^{19}$ atoms $\mathrm{cm}^{-2}$. The open circle (green) marks the position of Price-Whelan 1 , and the feature commonly attributed to the LA is indicated in the top panel.

We therefore take a closer look at the H I gas in the vicinity of Price-Whelan 1 to assess its possible relation to the MS. While no bulk metallicity measurements exist for the LA gas, recent measurements of oxygen and silicon abundances $([\mathrm{O} / \mathrm{H}] \sim-1)$ suggest that the gas has a comparable abundance pattern to the SMC gas (Fox et al. 2013, 2018; Richter et al. 2018). If Price-Whelan 1 formed from this gas, we would then expect that the stellar metallicity should be similar to young stars in the SMC. In the outskirts of the SMC, the metallicity distribution of the youngest stars (age $<2 \mathrm{Gyr}$ ) has a peak around $[\mathrm{Fe} / \mathrm{H}] \sim-1$ and a spread of $\sigma_{[\mathrm{Fe} / \mathrm{H}]} \sim$ 0.2 dex, which at least means that the metallicity of PriceWhelan $1([\mathrm{Fe} / \mathrm{H}] \approx-1.1)$ is consistent with young SMC stars (Dobbie et al. 2014).

We next look at the kinematics of high velocity gas in this region. Figure 7 shows the H I column density of the catalog of Gaussian centers (produced with the techniques and software from Nidever et al. 2008) from the Galactic All Sky Survey (GASS; McClure-Griffiths et al. 2009; Kalberla et al. 2010) in the region around the LA and plotted in the MS coordinate system ( $L_{\mathrm{MS}}, B_{\mathrm{MS}}$; Nidever et al. 2008). The location of Price-Whelan $l$ is marked (green circle), and the three panels show three different velocity slices (indicated on each panel). The MS gas is most visible and densest at the highest velocities where the LA II feature is found, around $L_{\mathrm{MS}} \lesssim 61^{\circ}$ and $B_{\mathrm{MS}} \sim-12^{\circ}$, but the lower

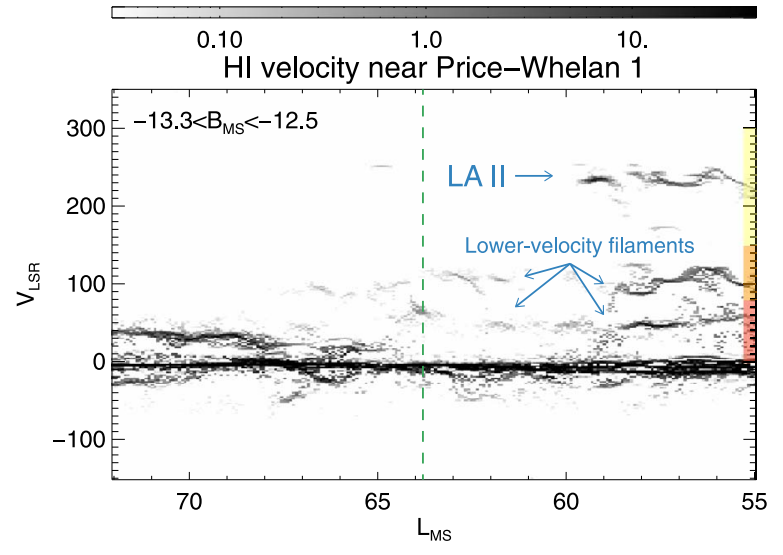

Figure 8. The position-velocity diagram of GASS H I Gaussian centers in a slice of Magellanic latitudes (indicated) in the same region as Figure 7, showing the LOS velocity (in the local standard of rest frame) of gas near Price-Whelan 1. The colored rectangles (red, orange, yellow) indicate the three velocity slices used in Figure 7 . Here, the colorbar shows the gas density in units of $10^{18}$ atoms $\mathrm{cm}^{-2} \mathrm{deg}$. Note that the densest gas at the longitude of Price-Whelan 1 (vertical line) appears to be associated with the lower velocity filaments seen at lower longitudes $L_{\mathrm{MS}} \lesssim 60^{\circ}$, which may be decelerated gas associated with the MS.

panels (i.e., slower gas) still displays some of the same morphology as the highest velocity slice.

This is also apparent in a position-velocity diagram of the same range of longitudes: Figure 8 shows the velocity of gas in this region (all longitudes, but near $B_{\mathrm{MS}} \sim-13^{\circ}$ ) as a function of $L_{\mathrm{MS}}$. Here, the longitude of Price-Whelan 1 is marked as the dashed vertical (green) line. The LA II feature is again visible as the $V_{\mathrm{LSR}}>200 \mathrm{~km} \mathrm{~s}^{-1}$ filament of gas at $L_{\mathrm{MS}} \lesssim 60^{\circ}$ (and a faint extension near $L_{\mathrm{MS}} \sim 65^{\circ}$ ), but two other prominent filaments with similar morphologies are apparent at lower velocities $V_{\mathrm{LSR}} \sim 60 \mathrm{~km} \mathrm{~s}^{-1}$ and $V_{\mathrm{LSR}} \sim 100 \mathrm{~km} \mathrm{~s}^{-1}$ from $L_{\mathrm{MS}} \lesssim 65^{\circ}$.

The entire region shown in Figure 7 is on the other side of the Galactic disk with respect to the MCs, meaning that all of the MS gas in this region has passed through the Galactic midplane. The similar filamentary structure in the high and lower velocity gas therefore raises the question: Did the low velocity filaments originate in the MS, but were decelerated through interactions with Milky Way gas? This scenario has been suggested (i.e., that the LA material has interacted with gas in the Galactic disk) based on the kinematics and morphology of a LA HVC (McClureGriffiths et al. 2008) and presents a possible formation scenario for Price-Whelan 1. If the cluster formed from the LA gas, and some of the gas interacted with the Milky Way disk, the dynamics of the stars and the gas that interacted would have decoupled as soon as the stars formed. Given the geometry of the current location of Price-Whelan 1 (i.e., our viewing angle from the solar position), in this scenario we would expect a moderate velocity difference between the gas that interacted and stars to primarily appear as a difference in the tangential velocities of the stars and the gas (which is not measured). We can therefore use the LOS velocity of the gas as a proxy for the LOS velocity of the stars in Price-Whelan 1 to study the Galactic orbit of the cluster, as discussed in the next section, and because we do not yet have radial velocity information for the stars.

\subsection{The Galactic Orbit of Price-Whelan 1}

Since the gas distribution in Figure 8 shows excess at velocities $V_{\mathrm{LSR}} \approx(60,110,230) \mathrm{km} \mathrm{s}^{-1}$ near the location of 

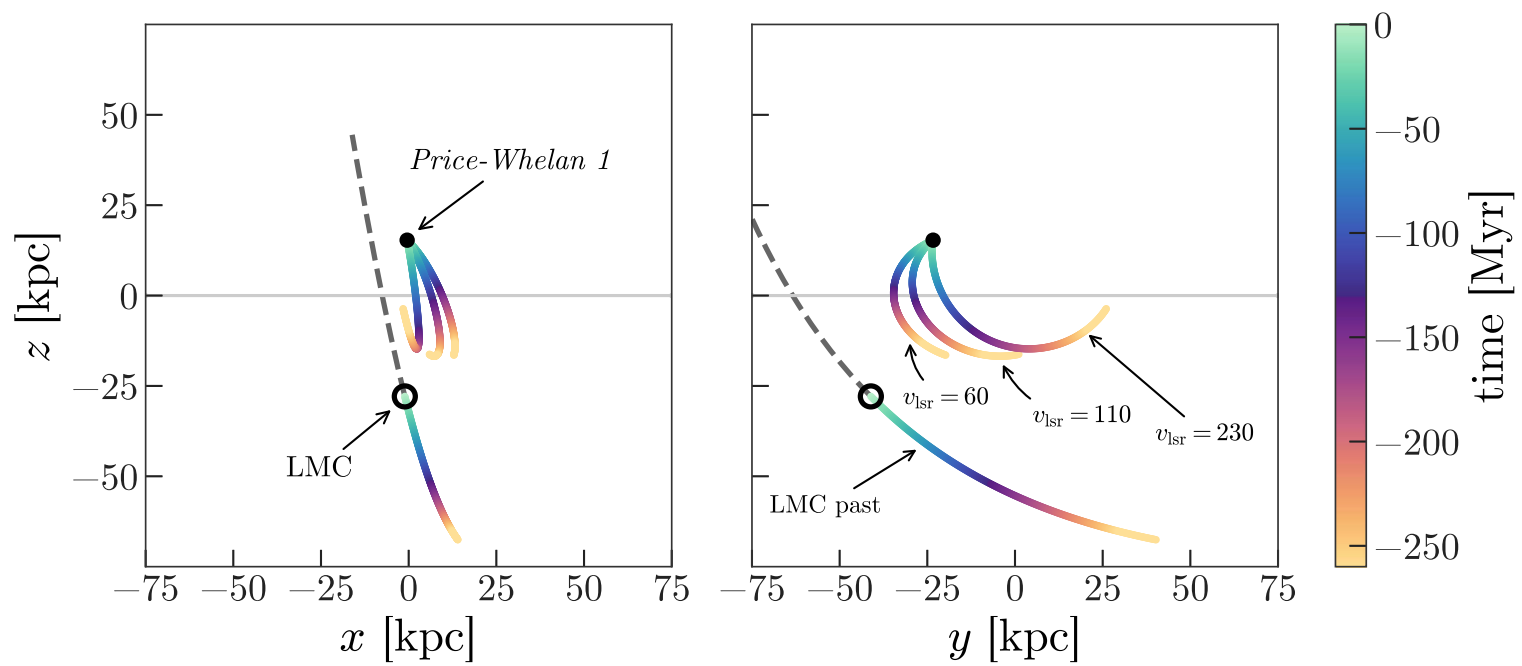

Figure 9. The past orbit of Price-Whelan 1 in Galactocentric Cartesian coordinates, computed by assuming an LOS velocity equal to each of three different possible gas filaments in the LA II region of the MS (as indicated in right panel). In these coordinates, the Sun is at $(x, y, z)=(-8.1,0,0) \mathrm{kpc}$, and the present-day locations of Price-Whelan 1 (filled black marker) and the LMC (open black marker) are indicated. The time at each point in the orbits is indicated by the colorbar, with the darkest regions corresponding to $t=-130 \mathrm{Myr}$, the inferred age of Price-Whelan 1. The past and future orbit of the LMC are shown as the colored line and dashed line, respectively.

the cluster (ignoring the disk gas between $V_{\mathrm{LSR}} \sim-20$ and $20 \mathrm{~km} \mathrm{~s}^{-1}$ ), it seems that there are three qualitatively different LOS velocities that the gas (and plausibly Price-Whelan 1) could have. If we adopt these velocity measurements as the LOS velocity of Price-Whelan 1, we have measurements of all six phase-space components and can compute orbits for the cluster. We compute Galactic orbits of the cluster using each of the three values of the LOS velocity from the LA II region filaments. Figure 9 shows these three orbits computed by integrating the position of Price-Whelan 1 backwards in time: the two panels of Figure 9 show the Galactocentric Cartesian trajectories of PriceWhelan 1 over the last $260 \mathrm{Myr}$ by assuming each of the three plausible LOS velocities discussed above (as labeled in the right panel). To compute the orbits, we use a three-component Milky Way mass model consisting of a disk (Miyamoto \& Nagai 1975), a bulge (Hernquist 1990), and a spherical dark matter halo (Navarro et al. 1996). The parameters of this model are set to match the circular velocity profile and disk properties of Bovy (2015), and it is implemented in the gala package (PriceWhelan 2017) as the MilkyWayPotential.

We tried including an LMC component as a Hernquist sphere (Hernquist 1990) with masses between $10^{10}$ and $2.5 \times 10^{11} M_{\odot}$, but find that the orbit of Price-Whelan 1 does not change much over the integration period. If the LMC mass were as high as $8 \times 10^{11} M_{\odot}$, all three orbits of Price-Whelan 1 return to and end up bound to the LMC, but at this large of a mass, the assumption of a fixed Milky Way reference frame is invalid. We therefore neglect LMC-SMC component in what follows, and present an alternate interpretation below.

Also shown in Figure 9 is the present-day location of the LMC (open black circle), the present-day location of PriceWhelan 1 (filled black circle), the past orbit of the LMC over the same period, and the projected future orbit of the LMC (dashed line), all excluding the LMC-SMC as a mass component. For the two lower LOS velocity cases, the orbit of Price-Whelan 1 crosses the midplane of the Galactic disk at a time comparable to the age of the cluster (i.e., when the color is darkest, corresponding to a time $\approx-130 \mathrm{Myr})$. However, while the orbits of Price-Whelan 1 and the LMC are generally in the same orbital plane and have the same sense about the Galaxy, none of the orbits closely approach (within the halfmass-radius of our Hernquist model for the LMC) or cross the orbit of the LMC (even when including the LMC mass component). This is expected if the gas was shocked or otherwise lost kinetic energy through, e.g., ram pressure-as is expected to explain the morphology of the LA material (e.g., Hammer et al. 2015)_-during or before the star formation event that produced Price-Whelan 1. We therefore posit that the orbit of Price-Whelan 1 can be explained as having originated from the LMC-SMC system with some loss of orbital energy during the star formation event.

Precise radial velocity measurements of stars in PriceWhelan 1 will definitively rule on this scenario. For one, with a precision $\sigma_{v} \lesssim 2 \mathrm{~km} \mathrm{~s}^{-1}$, the internal kinematics of PriceWhelan 1 could be resolved, and in particular the expansion rate could be measured. But even low-precision velocity measurements would either confirm the association between Price-Whelan 1 and one of the three LA II-region gas filaments, or would challenge this scenario.

\subsection{Other Possible Formation Scenarios}

While the spatial proximity of Price-Whelan 1 to LA gas in the MS suggests a tentative connection, radial velocity data for the stars is required to confirm that the velocity of the cluster is consistent with the gas and thereby confirm the connection (D. L. Nidever et al. 2019, in preparation). However, even if the cluster can be confidently, kinematically associated with the LA gas, a number of mysteries would remain. First, it is not clear what could have triggered this star formation event, or why this appears to be the only young cluster associated with the LA gas. Second, the origin of the LA gas itself is not well understood, and some studies have questioned its connection to the MCs (Tepper-García et al. 2019).

Other possible formation scenarios for Price-Whelan 1 do not help to resolve these issues. One possible alternative scenario is that the Price-Whelan 1 birth cloud was unassociated with the MS and instead a lone HVC. However, this still raises questions about what triggered the star formation, given that other HVCs in 
the Milky Way are observed to be devoid of stars (e.g., Stark et al. 2015). Another possible origin for Price-Whelan 1 is that it formed from gas stripped from a fully destroyed dwarf galaxy (an unknown satellite of the MCs). However, we have not found a significant over-density of comoving older stars associated with the LA in this region.

One final possibility is that these stars were formed in or associated with the Galactic disk. While there have been some claimed discoveries of young, embedded clusters at high Galactic latitude (within $\lesssim 5 \mathrm{kpc}$ of the Sun, e.g., Camargo et al. 2015, 2016, these may not be real Turner et al. 2017. Still, stars formed in the Galactic disk have been observed far from the disk midplane with heights up to $|z| \sim 10 \mathrm{kpc}$ e.g., the TriAnd over-density; Price-Whelan et al. 2015; Bergemann et al. 2018). However, TriAnd has an old stellar population, and no young disk stars have been since found associated with this or other features in the outer Galactic disk (Deason et al. 2018; Laporte et al. 2019).

\section{Summary}

We have identified a young, metal-poor stellar association in the Galactic halo-named Price-Whelan 1-with an age $\tau \approx 117 \mathrm{Myr}$, heliocentric distance $D \approx 28.7 \mathrm{kpc}$, and metallicity $[\mathrm{Fe} / \mathrm{H}] \approx-1.14$. At its present-day sky position, and at large distances, all significant quantities of $\mathrm{H} \mathrm{I}$ are associated with the leading arm of the MS, and thus it plausibly formed from this gas. The age of the cluster is broadly consistent with the time it would have most recently crossed the Galactic midplane, suggesting the possibility that interaction with the Milky Way disk or tidal compression could have triggered this star formation event. Previous studies have detected young stars in the leading arm and in the periphery of the LMC (Casetti-Dinescu et al. 2014; Moni Bidin et al. 2017); however, this is the first time that an entire young star cluster has been detected so far from the Clouds.

The discovery of Price-Whelan 1 provides a critical distance constraint to the leading arm MS and will aid future MS and Milky Way modeling efforts. It also provides an opportunity to study star formation in a unique environment (i.e., low gas density, low metallicity, and large velocity), unlike that of the Milky Way disk or any other Local Cluster-forming region. The serendipitous discovery of this cluster is a reminder that the combined value of the Gaia data with deep, large-area imaging surveys provides a wealth of information about our Galaxy and stellar halo.

We thank Doug Finkbeiner (Harvard) for contributing to the DECam observations that enabled this work.

It is a pleasure to thank Lauren Anderson (Flatiron), Ana Bonaca (Harvard), Elena D’Onghia (UW Madison), Dan Foreman-Mackey (Flatiron), Raja Guhathakurta (UCSC), Cliff Johnson (Northwestern), Semyeong Oh (Cambridge), Ekta Patel (Arizona), Sarah Pearson (Flatiron), Josh Peek (STScI), Anil Seth (Utah), and Erik Tollerud (STScI) for useful suggestions and discussion.

D.L.N. thanks Peter Kalberla for making the GASS data cubes available in MS coordinates.

S.K. is supported by National Science Foundation grant AST-1813881.

The DECam results are based on observations at Cerro Tololo Inter-American Observatory, National Optical Astronomy Observatory (NOAO Prop. ID: 2018A-0251; PI: Finkbeiner), which is operated by the Association of Universities for
Research in Astronomy (AURA) under a cooperative agreement with the National Science Foundation.

This work has made use of data from the European Space Agency (ESA) mission Gaia (https://www.cosmos.esa.int/ gaia), processed by the Gaia Data Processing and Analysis Consortium (DPAC, https://www.cosmos.esa.int/web/gaia/ $\mathrm{dpac} /$ consortium). Funding for the DPAC has been provided by national institutions, in particular the institutions participating in the Gaia Multilateral Agreement.

We thank the Scientific Computing Core at the Flatiron Institute, and especially Dylan Simon and Nick Carriero, for technical support and access to the Flatiron Institute cluster computing resources, which enabled this work. We thank the Center for Computational Astrophysics and especially David Spergel for support, access to computational resources, and space to conduct this work.

Facility: Blanco (DECam).

Software: Astropy (Astropy Collaboration et al. 2013, 2018), dustmaps (Green 2018), emcee (Foreman-Mackey et al. 2013b, 2013a), gala (Price-Whelan 2017), IPython (Pérez \& Granger 2007), isochrones (Morton 2015), matplotlib (Hunter 2007), numpy (Walt et al. 2011), PyMultinest (Buchner et al. 2014), schwimmbad (Price-Whelan \& Foreman-Mackey 2017), scipy (Jones et al. 2001).

\section{Appendix \\ Queries}

Initial query to select very blue stars away from the Galactic plane:

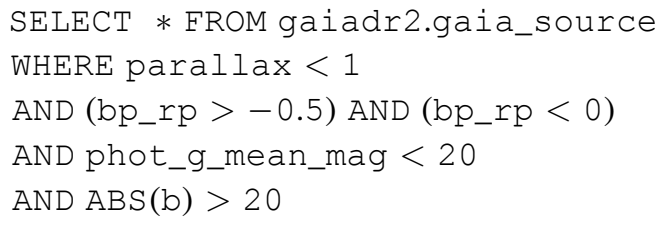

Query to retrieve Gaia data around the blue, comoving group found and discussed in Section 2:

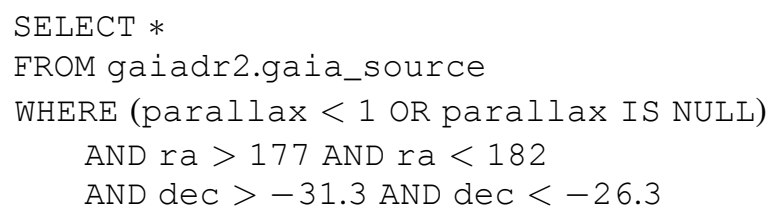

\section{ORCID iDs}

Adrian M. Price-Whelan (iD https://orcid.org/0000-00030872-7098

David L. Nidever (1) https://orcid.org/0000-0002-1793-3689 Yumi Choi (iD https://orcid.org/0000-0003-1680-1884 Edward F. Schlafly (D) https://orcid.org/0000-0002-3569-7421 Timothy Morton (iD https://orcid.org/0000-0002-8537-5711 Sergey E. Koposov (i) https://orcid.org/0000-0003-2644-135X Vasily Belokurov (iD https://orcid.org/0000-0002-0038-9584

\section{References}

Astropy Collaboration, Price-Whelan, A. M., Sipócz, B. M., et al. 2018, AJ, 156,123

Astropy Collaboration, Robitaille, T. P., Tollerud, E. J., et al. 2013, A\&A, $558, \mathrm{~A} 33$ 
Bekki, K., Chiba, M., \& McClure-Griffiths, N. M. 2008, ApJL, 672, L17 Belokurov, V. A., \& Erkal, D. 2019, MNRAS, 482, L9

Bergemann, M., Sesar, B., Cohen, J. G., et al. 2018, Natur, 555, 334 Besla, G., Kallivayalil, N., Hernquist, L., et al. 2007, ApJ, 668, 949 Besla, G., Kallivayalil, N., Hernquist, L., et al. 2010, ApJL, 721, L97 Besla, G., Kallivayalil, N., Hernquist, L., et al. 2012, MNRAS, 421, 2109 Bovy, J., Hogg, D. W., \& Roweis, S. T. 2011, AnApS, 5, 1657 Bovy, J. 2015, ApJS, 216, 29

Bregman, J. N. 1980, ApJ, 236, 577

Brüns, C., Kerp, J., Staveley-Smith, L., et al. 2005, A\&A, 432, 45

Buchner, J., Georgakakis, A., Nandra, K., et al. 2014, A\&A, 564, A125

Burton, W. B., \& Lockman, F. J. 1999, A\&A, 349, 7

Camargo, D., Bica, E., \& Bonatto, C. 2016, A\&A, 593, A95

Camargo, D., Bica, E., Bonatto, C., \& Salerno, G. 2015, MNRAS, 448, 1930

Carrera, R., Conn, B. C., Noël, N. E. D., Read, J. I., \& López Sánchez, Á R. 2017, MNRAS, 471, 4571

Casetti-Dinescu, D. I., Moni Bidin, C., Girard, T. M., et al. 2014, ApJL, 784, L37

Chambers, K. C., Magnier, E. A., Metcalfe, N., et al. 2016, arXiv:1612.05560

Choi, J., Dotter, A., Conroy, C., et al. 2016, ApJ, 823, 102

Choi, Y., Nidever, D. L., Olsen, K., et al. 2018a, ApJ, 866, 90

Choi, Y., Nidever, D. L., Olsen, K., et al. 2018b, arXiv:1805.00481

Danielski, C., Babusiaux, C., Ruiz-Dern, L., Sartoretti, P., \& Arenou, F. 2018, A\&A, 614, A19

Deason, A. J., Belokurov, V., \& Koposov, S. E. 2018, MNRAS, 473, 2428

Deason, A. J., Belokurov, V., \& Weisz, D. R. 2015, MNRAS, 448, L77

Diaz, J. D., \& Bekki, K. 2012, ApJ, 750, 36

Dobbie, P. D., Cole, A. A., Subramaniam, A., \& Keller, S. 2014, MNRAS, 442,1680

D’Onghia, E., \& Fox, A. J. 2016, ARA\&A, 54, 363

Dotter, A. 2016, ApJS, 222, 8

Feroz, F., \& Hobson, M. P. 2008, MNRAS, 384, 449

Feroz, F., Hobson, M. P., \& Bridges, M. 2009, MNRAS, 398, 1601

Fiorentino, G., Bono, G., Monelli, M., et al. 2015, ApJL, 798, L12

For, B. Q., Staveley-Smith, L., Matthews, D., \& McClure-Griffiths, N. M. 2014, ApJ, 792, 43

Foreman-Mackey, D., Conley, A., Meierjurgen Farr, W., et al. 2013a, emcee: The MCMC Hammer, Astrophysics Source Code Library, ascl:1303.002

Foreman-Mackey, D., Hogg, D. W., Lang, D., \& Goodman, J. 2013b, PASP, 125,306

Foreman-Mackey, D., Hogg, D. W., \& Morton, T. D. 2014, ApJ, 795, 64

Fox, A. J., Barger, K. A., Wakker, B. P., et al. 2018, ApJ, 854, 142

Fox, A. J., Lehner, N., Lockman, F. J., et al. 2016, ApJL, 816, L11

Fox, A. J., Richter, P., Wakker, B. P., et al. 2013, ApJ, 772, 110

Gaia Collaboration, Babusiaux, C., van Leeuwen, F., et al. 2018a, A\&A, 616, A10

Gaia Collaboration, Brown, A. G. A., Vallenari, A., et al. 2018b, arXiv:1804. 09365

Gaia Collaboration, Prusti, T., de Bruijne, J. H. J., et al. 2016, A\&A, 595, A1 Gelman, A., \& Rubin, D. B. 1992, StaSc, 7, 457

Goodman, J., \& Weare, J. 2010, Commun. Appl. Math. Comput. Sci., 5, 65

Gossage, S., Conroy, C., Dotter, A., et al. 2018, ApJ, 863, 67

Green, G. 2018, JOSS, 3, 695

Guhathakurta, P., \& Reitzel, D. B. 1998, in ASP Conf. Ser 136, Galactic Halos: A UC Santa Cruz Workshop, ed. D. Zaritsky (San Francisco, CA: ASP), 22

Hammer, F., Yang, Y. B., Flores, H., Puech, M., \& Fouquet, S. 2015, ApJ, 813,110

Harris, W. E. 1996, AJ, 112, 1487

Hernquist, L. 1990, ApJ, 356, 359

Hogg, D. W., Myers, A. D., \& Bovy, J. 2010, ApJ, 725, 2166

Hunter, J. D. 2007, CSE, 9, 90

Ibata, R. A., Gilmore, G., \& Irwin, M. J. 1994, Natur, 370, 194

Johnston, C., Tkachenko, A., Aerts, C., et al. 2019, MNRAS, 482, 1231

Jones, E., Oliphant, T., Peterson, P., et al. 2001, SciPy: Open Source Scientific Tools for Python v1.2.1, http://www.scipy.org/

Kalberla, P. M. W., McClure-Griffiths, N. M., Pisano, D. J., et al. 2010, A\&A, 521, A17
Kallivayalil, N., van der Marel, R. P., Alcock, C., et al. 2006, ApJ, 638, 772 Kallivayalil, N., van der Marel, R. P., Besla, G., Anderson, J., \& Alcock, C. 2013, ApJ, 764, 161

Kroupa, P. 2001, MNRAS, 322, 231

Laporte, C. F. P., Belokurov, V., Koposov, S. E., Smith, M. C., \& Hill, V. 2019, arXiv:1907.10678

Lindegren, L., Hernandez, J., Bombrun, A., et al. 2018, arXiv:1804.09366

Mackey, A. D., Koposov, S. E., Da Costa, G. S., et al. 2017, MNRAS, 472, 2975

Mackey, A. D., Koposov, S. E., Erkal, D., et al. 2016, MNRAS, 459, 239

Maíz Apellániz, J., \& Weiler, M. 2018, A\&A, 619, A180

Majewski, S. R., Skrutskie, M. F., Weinberg, M. D., \& Ostheimer, J. C. 2003, ApJ, 599, 1082

Mathewson, D. S., Cleary, M. N., \& Murray, J. D. 1974, ApJ, 190, 291

Mayer, L., Mastropietro, C., Wadsley, J., Stadel, J., \& Moore, B. 2006 MNRAS, 369, 1021

McClure-Griffiths, N. M., Pisano, D. J., Calabretta, M. R., et al. 2009, ApJS, 181,398

McClure-Griffiths, N. M., Staveley-Smith, L., Lockman, F. J., et al. 2008, ApJL, 673, L143

McConnachie, A. W. 2012, AJ, 144, 4

Miyamoto, M., \& Nagai, R. 1975, PASJ, 27, 533

Moni Bidin, C., Casetti-Dinescu, D. I., Girard, T. M., et al. 2017, MNRAS, 466, 3077

Morton, T. D. 2015, Isochrones: Stellar Model Grid Package, Astrophysics Source Code Library, ascl:1503.010

Navarro, J. F., Frenk, C. S., \& White, S. D. M. 1996, ApJ, 462, 563

Nidever, D. L., Majewski, S. R., \& Butler Burton, W. 2008, ApJ, 679, 432

Nidever, D. L., Majewski, S. R., Butler Burton, W., \& Nigra, L. 2010, ApJ, 723, 1618

Nidever, D. L., Olsen, K., Walker, A. R., et al. 2017, AJ, 154, 199

Noël, N. E. D., Conn, B. C., Carrera, R., et al. 2013, ApJ, 768, 109

Olsen, K. A. G., Zaritsky, D., Blum, R. D., Boyer, M. L., \& Gordon, K. D 2011, ApJ, 737, 29

Pardy, S. A., D’Onghia, E., \& Fox, A. J. 2018, ApJ, 857, 101

Paxton, B., Bildsten, L., Dotter, A., et al. 2011, ApJS, 192, 3

Paxton, B., Cantiello, M., Arras, P., et al. 2013, ApJS, 208, 4

Paxton, B., Marchant, P., Schwab, J., et al. 2015, ApJS, 220, 15

Pérez, F., \& Granger, B. E. 2007, CSE, 9, 21

Price-Whelan, A. M. 2017, JOSS, 2, 388

Price-Whelan, A. M., \& Foreman-Mackey, D. 2017, JOSS, 2, 357

Price-Whelan, A. M., Hogg, D. W., Rix, H.-W., et al. 2018, AJ, 156, 18

Price-Whelan, A. M., Johnston, K. V., Sheffield, A. A., Laporte, C. F. P., \& Sesar, B. 2015, MNRAS, 452, 676

Putman, M. E., Gibson, B. K., Staveley-Smith, L., et al. 1998, Natur, 394, 752

Richter, P., Fox, A. J., Wakker, B. P., et al. 2018, arXiv:1808.09455

Salpeter, E. E. 1955, ApJ, 121, 161

Schlafly, E. F., \& Finkbeiner, D. P. 2011, ApJ, 737, 103

Schlegel, D. J., Finkbeiner, D. P., \& Davis, M. 1998, ApJ, 500, 525

Smith, G. P. 1963, BAN, 17, 203

Stark, D. V., Baker, A. D., \& Kannappan, S. J. 2015, MNRAS, 446, 1855

Stetson, P. B. 1987, PASP, 99, 191

Stetson, P. B. 1994, PASP, 106, 250

Tepper-García, T., \& Bland-Hawthorn, J. 2018, MNRAS, 478, 5263

Tepper-García, T., Bland-Hawthorn, J., Pawlowski, M. S., \& Fritz, T. K. 2019, MNRAS, 488, 918

Turner, D. G., Carraro, G., \& Panko, E. A. 2017, MNRAS, 470, 481

Valdes, F., Gruendl, R. \& DES Project 2014, in ASP Conf. Ser. 485, Astronomical Data Analysis Software and Systems XXIII, ed. N. Manset \& P. Forshay (San Francisco, CA: ASP), 379

Venzmer, M. S., Kerp, J., \& Kalberla, P. M. W. 2012, A\&A, 547, A12

Walt, S. v. d., Colbert, S. C., \& Varoquaux, G. 2011, CSE, 13, 22

Wolf, C., Onken, C. A., Luvaul, L. C., et al. 2018, PASA, 35, e010

Yang, W., \& Tian, Z. 2017, ApJ, 836, 102

Zhang, L., Moni Bidin, C., Casetti-Dinescu, D. I., et al. 2017, ApJ, 835, 285

Zivick, P., Kallivayalil, N., van der Marel, R. P., et al. 2018, ApJ, 864, 55 